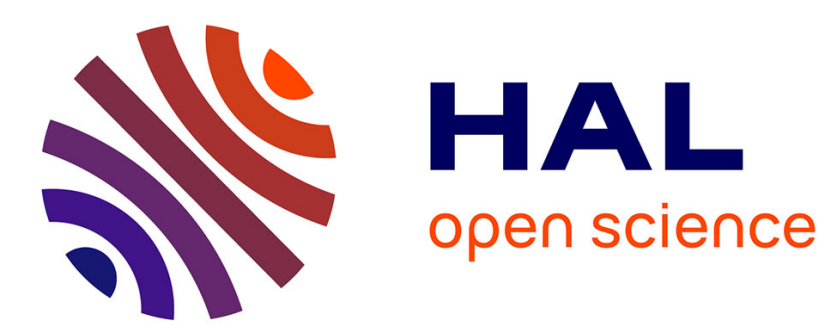

\title{
Cerebrospinal fluid volume analysis for hydrocephalus diagnosis and clinical research
}

\author{
Alain Lebret, Jérôme Hodel, Alain Rahmouni, Philippe Decq, Eric Petit
}

\section{To cite this version:}

Alain Lebret, Jérôme Hodel, Alain Rahmouni, Philippe Decq, Eric Petit. Cerebrospinal fluid volume analysis for hydrocephalus diagnosis and clinical research. Computerized Medical Imaging and Graphics, 2013, 37 (3), pp.224-233. 10.1016/j.compmedimag.2013.03.005 . hal-00813015

\section{HAL Id: hal-00813015 https://hal.science/hal-00813015}

Submitted on 31 May 2013

HAL is a multi-disciplinary open access archive for the deposit and dissemination of scientific research documents, whether they are published or not. The documents may come from teaching and research institutions in France or abroad, or from public or private research centers.
L'archive ouverte pluridisciplinaire HAL, est destinée au dépôt et à la diffusion de documents scientifiques de niveau recherche, publiés ou non, émanant des établissements d'enseignement et de recherche français ou étrangers, des laboratoires publics ou privés. 


\title{
Cerebrospinal fluid volume analysis for hydrocephalus diagnosis and clinical research
}

\author{
Alain Lebret ${ }^{\mathrm{a}}$, Jérôme Hodel ${ }^{\mathrm{b}}$, Alain Rahmouni ${ }^{\mathrm{c}}$, Philippe Decq ${ }^{\mathrm{c}}$, Éric Petit ${ }^{\mathrm{a}}$ \\ ${ }^{a}$ Université Paris-Est, LISSI (EA 3956), UPEC, F-94010, Créteil, France \\ ${ }^{b}$ Hôpital Saint-Joseph, 185 Rue Raymond Losserand, F-75014, Paris, France \\ ${ }^{c}$ Hôpital Henri Mondor, 51 Av du Maréchal de Lattre de Tassigny, F-94010, Créteil, France
}

\begin{abstract}
In this paper we analyze volumes of the cerebrospinal fluid spaces for the diagnosis of hydrocephalus, which are served as reference values for future studies. We first present an automatic method to estimate those volumes from a new three-dimensional whole body magnetic resonance imaging sequence. This enables us to statistically analyze the fluid volumes, and to show that the ratio of subarachnoid volume to ventricular one is a proportionality constant for healthy adults $(=10.73)$, while in range $[0.63,4.61]$ for hydrocephalus patients. This indicates that a robust distinction between pathological and healthy cases can be achieved by using this ratio as an index.
\end{abstract}

Keywords: cerebrospinal fluid, hydrocephalus, volume assessment, three-dimensional image analysis, computer-aided diagnosis

\section{Introduction}

Hydrocephalus is a neurological disorder involving an abnormal accumulation of the cerebrospinal fluid in the cavities of the brain known as cerebral ventricles. The central nervous system is completely surrounded by the fluid contained within these ventricles and the subarachnoid space. A ventricle enlargement will increase pressure on the brain, which can result in damage to brain tissues and several brain malfunctions. The fluid circulates from ventricles where it is produced to the subarachnoid space where it is resorbed into the circulatory system [1]. Figure 1 shows descriptive representations of the fluid pathway from ventricles to the subarachnoid space in a sagittal plane (left) and through the various encountered spaces as proposed in [2] (right). Hydrocephalus can be classified into noncommunicating and communicating depending on whether the fluid pathway is obstructed or not [2]. The common test to diagnose hydrocephalus is requiring imaging techniques, such as computed tomography, magnetic resonance imaging, or pressure monitoring techniques in order to identify the enlarged ventricles and any obstruction in the fluid pathway. In routine clinical practice, those techniques are mainly limited to the intracranial region and may need

Email address: Alain-Rene.Lebret@ac-paris.fr (Alain Lebret) 


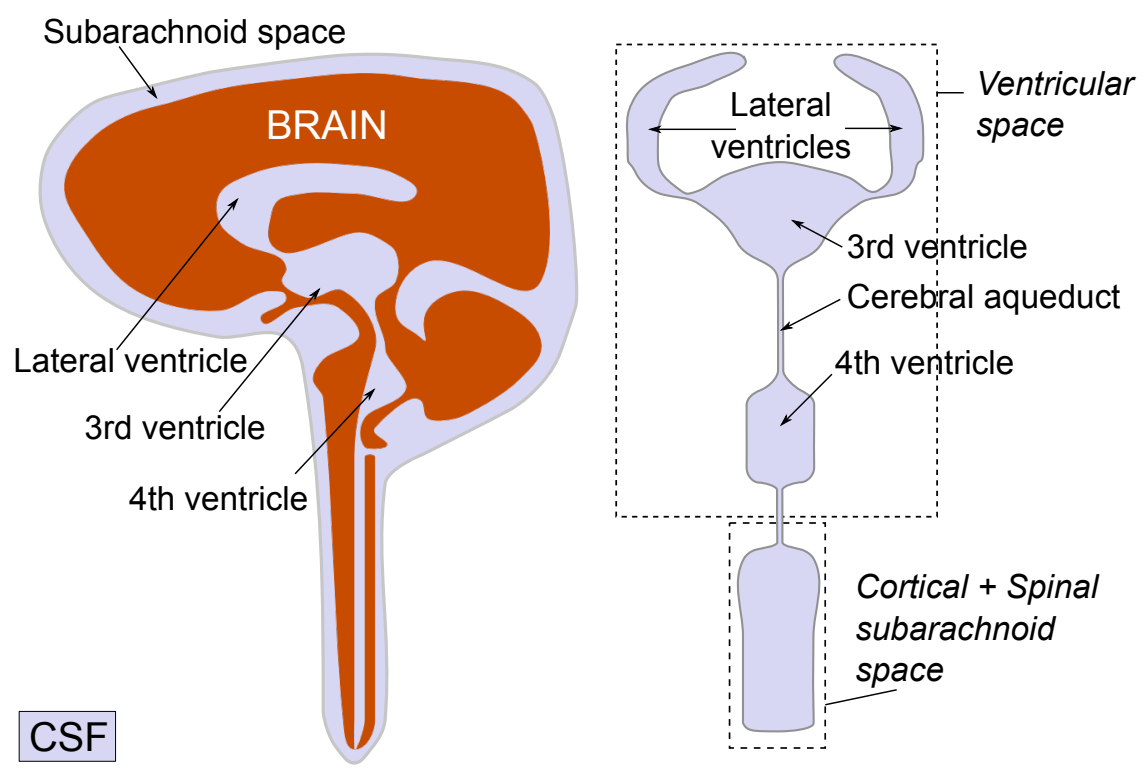

Figure 1: The cerebrospinal fluid circulates from ventricles where it is produced to the subarachnoid space where it is resorbed (left). The fluid flows through the various encountered spaces from the ventricles to the cortical subarachnoid space as proposed in [2] (right).

different sequences to identify the enlargement and highlight an obstruction. For instance, a ventricle enlargement can be identified by a magnetic resonance imaging sequence, while detecting a fluid flow malfunction may require an additional cine phase-contrast magnetic resonance imaging sequence.

Several papers have suggested a cerebrospinal fluid volume assessment to characterize hydrocephalus. For instance, a quantification is performed for healthy volunteers and patients with various types of hydrocephalus in [3, 4], whereas it is limited to normal pressure hydrocephalus, i.e. a specific case of communicating hydrocephalus in [5]. Nevertheless, all of them are restricted to the intracranial region, while it is assumed that the spinal subarachnoid space volume may also provide some information in hydrocephalus analysis [6]. Images reveal most cerebral structures, which do not facilitate segmentation of the fluid itself in a routine way. Thus, there is not yet any whole body method that is routinely used.

The aim of this work is to provide both a method to assess volumes of the entire cerebrospinal fluid in order to diagnose hydrocephalus and reference values for future studies. We have recently developed a new magnetic resonance imaging sequence that significantly highlights the cerebrospinal fluid [7] and proposed an automatic segmentation of its entire volume in [8]. To quantify the fluid volumes from those whole body images, we propose in this paper to improve the method described in [8] by automatically separating the entire fluid volume into its spinal subarachnoid, cortical subarachnoid and ventricular spaces. We also show the ability of our volume assessment to be exploited to perform statistical analyses, which lead to a functional parameter calculated by the ratio of the entire subarachnoid space volume to the ventricular one. We assume this parameter could be efficient to dis- 
criminate healthy adults from patients affected by communicating and non-communicating hydrocephalus. Furthermore, our approach only requires a single imaging sequence and an automatic image processing for each patient.

Concerning our segmentation method, we should consider the complex geometry of the cerebrospinal shape and its human variability, the presence of noise and artifacts, especially motion artifacts, and the possible obstructed fluid pathway, which results in a disconnection of the fluid spaces in non-communicating hydrocephalus cases. To overcome these problems, we introduce a topological assumption on the entire cerebrospinal fluid shape. This property, used as a refinement constraint, brings both accuracy and robustness to our segmentation method, particularly for pathological cases. The separation method of the previously segmented total fluid volume involves a region of interest defined from eyeballs. Spinal and intracranial spaces are separated by detecting a topological change of the fluid in the bottom part of the region of interest. Then, the ventricular space is separated from the intracranial space by reconstructing the ventricles from a specific tubular structure. All these steps are configured with default values that we specified, and can be chained without requiring any user interaction. Some steps may be even parallelized (e.g. the two separation methods).

This paper is organized as follows. Section 2 describes the segmentation method for the entire cerebrospinal fluid volume assessment. The separation of the entire volume into its ventricular and subarachnoid volumes is presented in Section 3. In Section 4, we first evaluate our volume assessment and then make statistical analyses on our clinical dataset, such as linear regression and linear discriminant analysis. Concluding remarks are given in Section 5 .

\section{Segmentation of the entire cerebrospinal fluid}

Our input whole body images appear to be binary due to the poor dynamic of the luminance perception of the human visual system but in fact they are not (see Fig. 2-a). Furthermore, images are disturbed by noise and artifacts. We thus use image properties and a topological assumption on the fluid shape for segmenting the entire cerebrospinal fluid volume.

\subsection{Topological model of the cerebrospinal fluid}

Topology is the mathematical study of spatial objects that are preserved through deformations such as twistings and stretchings, without regarding to their size and position. Properties of objects are considered by abstracting their inherent connectivities while ignoring their detailed shapes. More details on topology for digital images may be found in Chapter 6 of [9].

Numerous studies have demonstrated the effectiveness to integrate such topological constraints in medical image processing (see [10] for an overview). For instance, most brain structures have the topology of a filled or hollow sphere while their geometry is complex. We consider the entire fluid to be topologically equivalent to a filled sphere that is compliant with the fluid flow as shown in Fig. 1, while many people use rather a hollow sphere (or 
a more complex object) as its topological model [11, 12, 13]. Models closest to the reality $[11,13]$ are well adapted to address problems such as surface correction. However, our assumption significantly simplifies and also speeds up the initialization step compared to that of a hollow sphere, while the difference between the two resulting volumes is negligible. Furthermore, it introduces neither unexpected cavity nor handle despite of noise and artifacts, and is also more efficient and consistent for hydrocephalus patients for whom the fluid do not fit a hollow sphere anymore.

\subsection{Segmentation of the entire cerebrospinal fluid volume}

Segmentation consists in homotopically thicken an inner object $\left(C_{\text {inner }}\right)$, which is within the entire cerebrospinal fluid and is topologically equivalent to a filled sphere. This transformation, which preserves the topology of the inner object, is guided by a priority function based on a distance criterion. The inner object can be interactively or automatically defined, as long as it has the correct topology.

To significantly speed up the processing time, we propose to automatically initialize the inner object as close as possible to the border of the cerebrospinal fluid. We also initialize an outer object of the cerebrospinal fluid $\left(C_{\text {lim }}\right)$, which includes the entire fluid but do not necessarily have the same topology. Thus, guiding the thickening by the priority function is limited to the region between $C_{\text {inner }}$ and $C_{\text {lim }}$.

\subsubsection{Initialization of inner and outer objects}

An inner object $C_{\text {inner }}$ is initialized by applying the moment-preserving thresholding described in [14] on the initial image, followed by a larger connected component extraction (Fig. 2b). The moment-preserving thresholding is suited to "binary nature" images with low contrast. The moment preservation assumes that statistical moments on initial and thresholded images are identical. This method becomes more efficient than conventional clustering-based or entropy-based methods when the histogram presents an excessive overlapping between classes. Since the thresholded image contains several objects, a connected component labeling [15] is performed to extract the three largest connected components, which generally correspond to the inner object $C_{\text {inner }}$ and both eyeballs $E_{1}$ and $E_{2}$. $E_{1}$ and $E_{2}$ are retained to determine a region of interest in Section 3.1. By extracting the largest components, residual noise as well as other small objects such as salivary ducts are also removed. If $C_{\text {inner }}$ has holes, (see [9, Section 5.3.3] for a hole characterization) then the threshold is automatically adjusted by increasing its value until the topological assumption on $C_{\text {inner }}$ is satisfied; $E_{1}$ and $E_{2}$ are not affected by this operation due to their intensity.

To initialize an outer object $C_{\text {lim }}$, another histogram-based thresholding method is carried out on the initial image as described in [16]. The triangle thresholding algorithm in [16] is suitable for bimodal images for which the object to extract has a small amplitude and a large variance relatively to the background. It is followed by a largest connected component extraction to retrieve an outer object of the fluid $\left(C_{\lim }\right)$ as shown in Fig. 2c.

\subsubsection{Priority function}

The priority function $\Psi=\left\{\psi_{x}\right\}$ controls the order in which voxels are processed and eventually added to $C_{\text {inner }}$. This function uses an Euclidean distance map denoted by $D=$ 
$\left\{d_{x}\right\}$, which is defined for each voxel $x \in \bar{C}_{\text {inner }}$ as the distance to the nearest voxel of $C_{\text {inner }}$; it is calculated by the linear algorithm proposed in [17]. The value $\psi_{x}$ of each voxel $x$ in the outer object $C_{\lim }$ is then given by:

$$
\psi_{x}= \begin{cases}d_{x} & \text { if } x \in C_{\text {lim }} \\ +\infty & \text { otherwise }\end{cases}
$$

Euclidean distance transform was preferred to others because it improves selection of points that should be tested while the thickening process.

\subsubsection{Guided homotopic thickening}

Guided by the previous priority function $\Psi$, an homotopic thickening is applied to the inner object $C_{\text {inner }}$ by adding simple points.

A point is simple if its addition to or its removal from a binary object $C$ does not change the topology of the object and of the background. Therefore, removing simple points does not change the number of connected components and holes (cavities and/or handles) of $C$ and its complement $\bar{C}$. It is shown in [18] that a simple point $x$ is locally characterized by two local topological numbers. An efficient computation of these numbers that only involves the 26-neighborhood is also described in [18].

Let $\partial \bar{C}_{\text {inner }}$ denote the boundary of $C_{\text {inner }}$ such that $\partial \bar{C}_{\text {inner }}=\left\{x \in \bar{C}_{\text {inner }} \cap C_{\text {lim }} \mid N_{6}(x) \cap\right.$ $\left.C_{\text {inner }} \neq \emptyset\right\}$, where $N_{6}(x)$ is the 6 -neighborhood of $x$. The thickening process consists in adding to $C_{\text {inner }}$ simple points from $\partial \bar{C}_{\text {inner }}$ as presented in Algorithm 1 . It shows that boundary points (i.e. voxels) of $\partial \bar{C}_{\text {inner }}$ are chosen iteratively, according to their priorities, and are incorporated into $C_{\text {inner }}$ if they are simple.

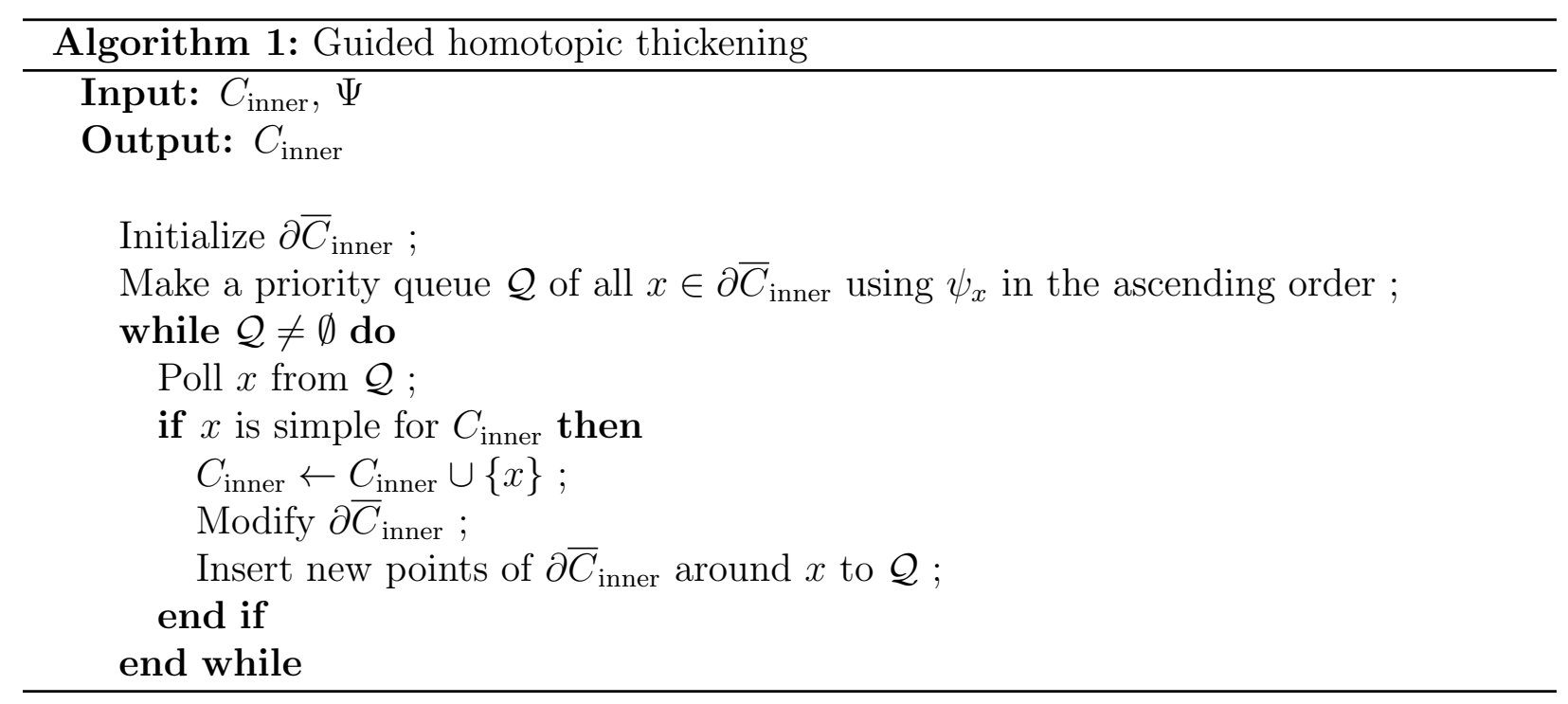

The resulting thickened object has the same topology as $C_{\text {inner }}$. It is illustrated in Fig. 2 d. 

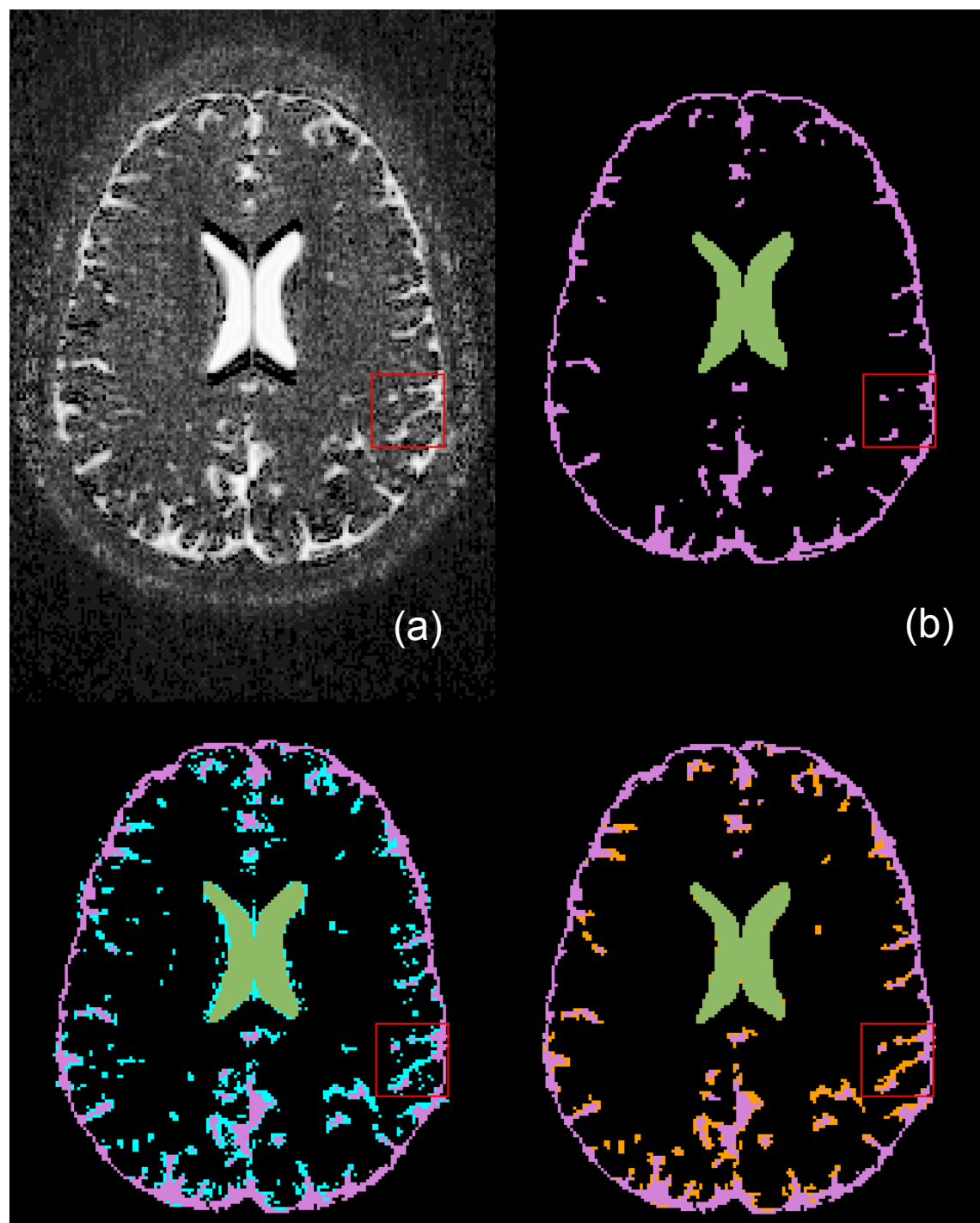

(c)

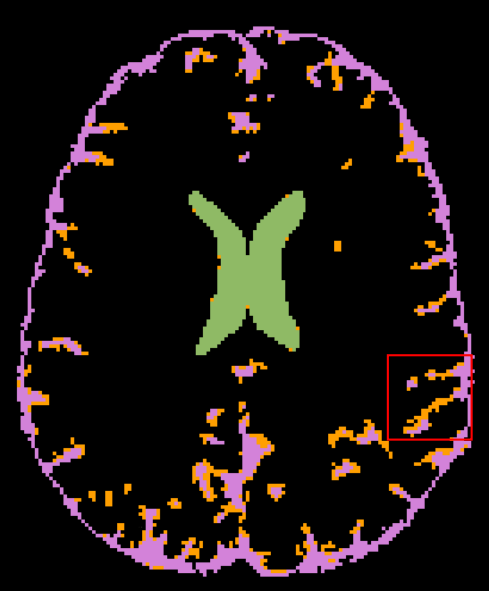

(d)

Figure 2: Segmentation process (axial cross-section), which is easily observed in the red square region. (a) contrast-enhanced original image; (b) inner object image $C_{\text {inner }}$ (ventricular and cortical subarachnoid spaces are represented respectively in green and magenta); (c) outer region image $C_{\lim }$ (voxels of $C_{\lim } \backslash C_{\text {inner }}$ are represented in cyan); (d) homotopic thickened image (voxels added to $C_{\text {inner }}$ are represented in orange). 


\section{Separation into cerebrospinal fluid volumes}

Separation of the cerebrospinal fluid spaces is required to analyze the relationship between the entire subarachnoid and ventricular volumes. First, the spinal and intracranial regions are separated using a topological property of the cerebrospinal fluid at their interface. Second, to retrieve the volume of the ventricular space from that of the cortical subarachnoid one, the cerebral aqueduct must be detected because we use it as a morphological reconstruction marker.

\subsection{Separation of spinal and intracranial spaces}

Expert physicians usually perform the disconnection of the spinal and intracranial spaces by tracking the foramen magnum (the hole in the bottom of the skull through which the spinal cord passes in order to be connected to the brain), which is, even for an expert, difficult to detect on this type of image. The separation into spinal and intracranial spaces requires to locate the closest axial cross-section plane to that of the foramen magnum. Thus, the disconnection is achieved by browsing the planar connected component on each axial plane from the spinal part, and by detecting any change in its topology (see the axial crosssection in Fig. 3). A region of interest is first determined to reduce the processing time as well as to improve the robustness.

\subsubsection{Determination of the region of interest}

A region of interest $\mathcal{R}$ is used to retrieve the cerebral aqueduct in order to separate the intracranial space into its ventricular and subarachnoid spaces (Section 3.2). Apart from pathological cases, eyeballs typically face the cerebral aqueduct in the axial plane. We consider the region forming a rectangular cuboid, each of whose edge is parallel to one of the $i-, j-, k$-axes of the image, such that:

- the region is located in the $i$-axis direction between the coronal plane that passes through the maximum posterior boundary of eyeballs, and the last coronal plane that still contains non-zero voxels;

- setting $D$ as the larger diameter of both eyeballs, the height of the region is set empirically to be $8 D$, such that the top face of the region is located with the distance $2 D$ above the eyeballs and the bottom face is located with the distance $5 D$ below the eyeballs. The height is chosen in agreement with an expert so as to contain all the necessary structures for the separation step. It may also be interactively resized to fit human variations;

- the depth is determined by the two sagittal planes between the medial boundaries of eyeballs.

\subsubsection{Determination of the cut-off plane}

Planar connected components are counted on each axial plane from the bottom to the top of the region of interest $\mathcal{R}$. The first axial plane that shows a change in the number of connected components is the plane just above that of the searched cut-off (Fig. 3). 

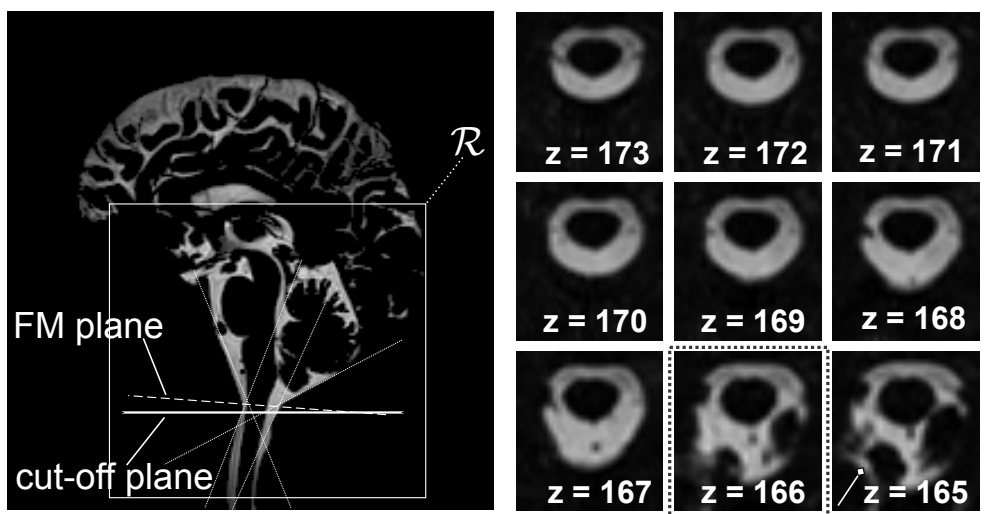

Figure 3: Separation of intracranial and spinal regions in the region of interest $\mathcal{R}$. The resulting cut-off plane $(z=166)$ is close to that goes through the foramen magnum (FM plane). The axial cross-sections around the cut-off plane are also illustrated (right).
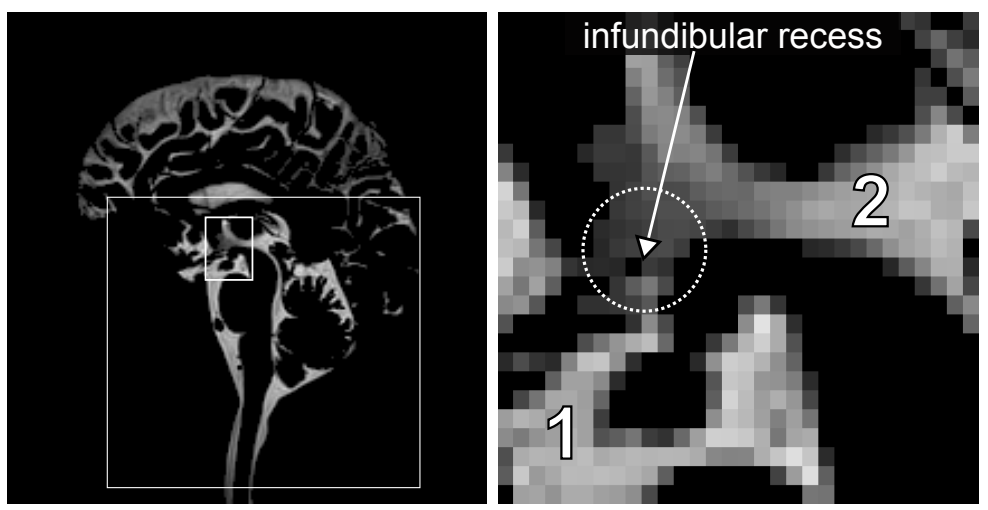

Figure 4: Proximity of the interpeduncular cistern (1) to the infundibular recess of the third ventricle (2). The small rectangular part in the left figure is zoomed in the right figure.

\subsection{Intracranial volume separation}

The process to extract the ventricular space may fail because of some subarachnoid regions that are very close to the third and fourth ventricles. Figure 4 shows, for instance, the proximity between the interpeduncular cistern and the infundibular recess of the third ventricle.

To overcome this problem we first detect the cerebral aqueduct that is a thin tubular structure (diameter: 1 to $3 \mathrm{~mm}$; length: around $14 \mathrm{~mm}$ ) located in the median sagittal plane, which connects the third and fourth ventricles. It is also the longest tubular structure with the highest intensity range in the most median sagittal planes of the region of interest $\mathcal{R}$. Its enhancement is performed by a vessel segmentation method (see [19]). Finally, ventricles are recovered by a morphological reconstruction [20] using the cerebral aqueduct as a marker.

\subsubsection{Detection of the cerebral aqueduct}

Several methods to enhance vessel like structures in grayscale images are reviewed in [19]. Several efficient methods are based on properties of eigenvalues of the Hessian matrix 
$\mathcal{H}[21,22]$. These multi-scale methods operate in a Gaussian scale space on which they calculate second-order derivatives, build the Hessian matrix $\mathcal{H}$, and decompose it depending on its eigenvalues $\lambda_{1}, \lambda_{2}$ and $\lambda_{3}$ such that $\left|\lambda_{1}\right|<\left|\lambda_{2}\right|<\left|\lambda_{3}\right|$. The eigenvalues are analyzed to determine the likelihood for each voxel $x$ to belong to a curvilinear structure. This analysis is based on the following assumptions: (1) $\lambda_{1} \approx 0$; (2) $\lambda_{2} \approx \lambda_{3}<0$; and (3) $\left|\lambda_{1}\right| \ll\left|\lambda_{2}\right|$.

The tubular structure filter implemented in [22] allows to dissociate strict tubular structures from others. We use it here to enhance the cerebral aqueduct. Let $F_{\sigma}=\left\{f_{x}^{\sigma}\right\}$ the filter in [22] such that:

$$
f_{x}^{\sigma}=\left\{\begin{array}{l}
0 \quad \text { if } \lambda_{2}>0 \text { or } \lambda_{3}>0, \\
\left(1-e^{-\frac{\mathcal{R}_{A}^{2}}{2 \alpha^{2}}}\right) e^{-\frac{\mathcal{R}_{B}^{2}}{2 \beta^{2}}}\left(1-e^{-\frac{\mathcal{S}^{2}}{2 c^{2}}}\right) \text { otherwise, }
\end{array}\right.
$$

where $\mathcal{R}_{A}=\left|\lambda_{2}\right| /\left|\lambda_{3}\right|, \mathcal{R}_{B}=\left|\lambda_{1}\right| / \sqrt{\left|\lambda_{2} \lambda_{3}\right|}, \mathcal{S}=\sqrt{\lambda_{1}^{2}+\lambda_{2}^{2}+\lambda_{3}^{2}}$, with some weights $\alpha \in$ $[0,1], \beta \in] 0,1]$ and $c \in[0,500]$. Figure 5 compares extracted curvilinear structures in the region of interest $\mathcal{R}$ using methods from [21] (Fig. 5b) and [22] (Fig. 5c). It shows that in contrast with [21], the filter in [22] allows to dissociate strict tubular structures from others. The cerebral aqueduct part has more highlighted values in Fig. $5 \mathrm{c}$ by adjusting the filter with a higher weight to detect lines $(\alpha=0.7)$. The aqueduct is the longest connected component found in the most median sagittal planes.

\subsubsection{Ventricles reconstruction}

Considering the cerebral aqueduct part as the marker, we aim to reconstruct the ventricular space. For this problem, methods such as fast marching [23], level set [23] or active contour models [24] are well known. However, the following two problems may hinder the reconstruction by the above methods: 1 ) the cerebral aqueduct part is too small as a marker to initialize a model; 2) motion artifacts can generate significant intensity inhomogeneities even in the ventricular space.

Geodesic reconstruction [20] is a very useful operator from mathematical morphology that provides quite satisfactory results here.

Let $\delta_{f, B_{\mathrm{d}}}^{(1)}(g)$ be the elementary geodesic dilation of a grayscale image $g$ inside $f(g$ is called the marker image and $f$ is the mask) such that $\delta_{f, B_{\mathrm{d}}}^{(1)}(g)=\left(g \oplus B_{\mathrm{d}}\right) \wedge f$, where $\wedge$ stands for the point-wise minimum and $g \oplus B_{\mathrm{d}}$ is the geodesic dilation of $g$ by an isotropic structuring element $B_{\mathrm{d}}$ chosen as a flat ball of radius $r_{d}=1$. The geodesic dilation of size $n \geq 0$ is obtained by:

$$
\delta_{f, B_{\mathrm{d}}}^{(n)}(g)=\underbrace{\delta_{f, B_{\mathrm{d}}}^{(1)} \circ \delta_{f, B_{\mathrm{d}}}^{(1)} \circ \cdots \circ \delta_{f, B_{\mathrm{d}}}^{(1)}(g)}_{n \text { times }} .
$$

The grayscale reconstruction by dilation $\rho_{f, B_{\mathrm{d}}}(g)$ of $f$ from $g$ is calculated by iterating geodesic dilations of $g$ inside $f$ until idempotence such that:

$$
\rho_{f, B_{\mathrm{d}}}(g)=\underset{n \geq 1}{\vee} \delta_{f, B_{\mathrm{d}}}^{(n)}(g)
$$

where $\vee$ stands for the point-wise maximum. 

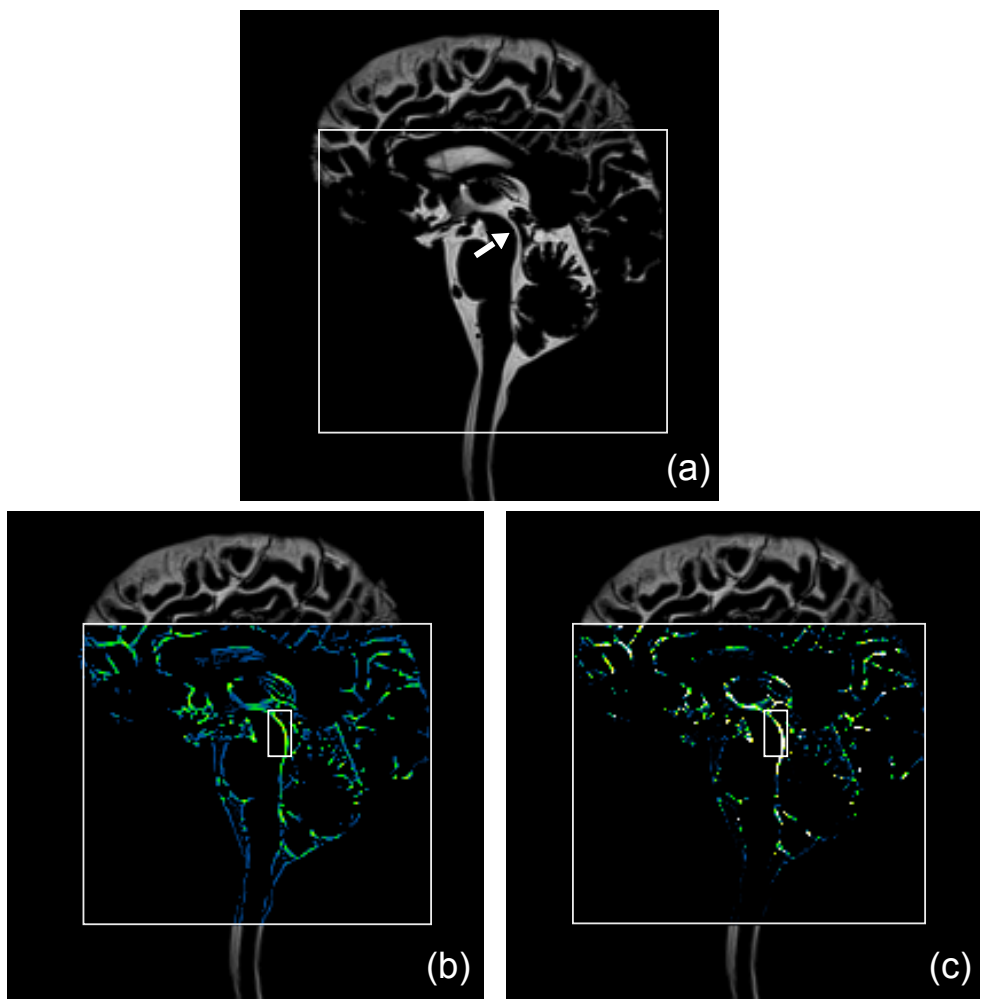

Figure 5: Detection of the cerebral aqueduct in the region of interest $\mathcal{R}$ (median plane crosssection). From left to right: (a) original thickened image; (b) filtered image by [21] $(\sigma=0.5)$; (c) filtered image by [22] using 5 scale spaces $\sigma$ from 0.4 to 0.9 and with $\alpha=0.7, \beta=0.2$ and $c=200$.

The geodesic reconstruction of the previously detected cerebral aqueduct used as the marker image is restricted to the region of interest. It generates an image composed of regional maxima due to the threshold decomposition used in [20]. The ventricular space is the union of the regional maxima whose intensities are greater than or equal to those which constitute the cerebral aqueduct. An $h$-dome transform [20] allows us to only keep these regional maxima. Figure 6 shows the result of such a reconstruction for an healthy adult by using a flat ball of radius $r_{d}=1$. Figure 7 shows a three-dimensional visualization of a part of the cerebrospinal fluid spaces obtained by our method; an healthy adult (left) and a non-communicating hydrocephalus patient (right).

\section{Cerebrospinal fluid volume analysis}

We first present experiments we have carried out to assess volumes of the cerebrospinal fluid spaces from whole body magnetic resonance images in two stages: 1) the segmentation method has been tested on phantoms of known volume; 2) the segmentation and separation steps have been performed on a clinical dataset. 12 healthy controls and 26 patients have been processed; no manual intervention was required for any of them.

We then statistically analyze the distribution of the subarachnoid space volumes relative to the ventricular ones. More specifically we apply a linear regression on the healthy adults 

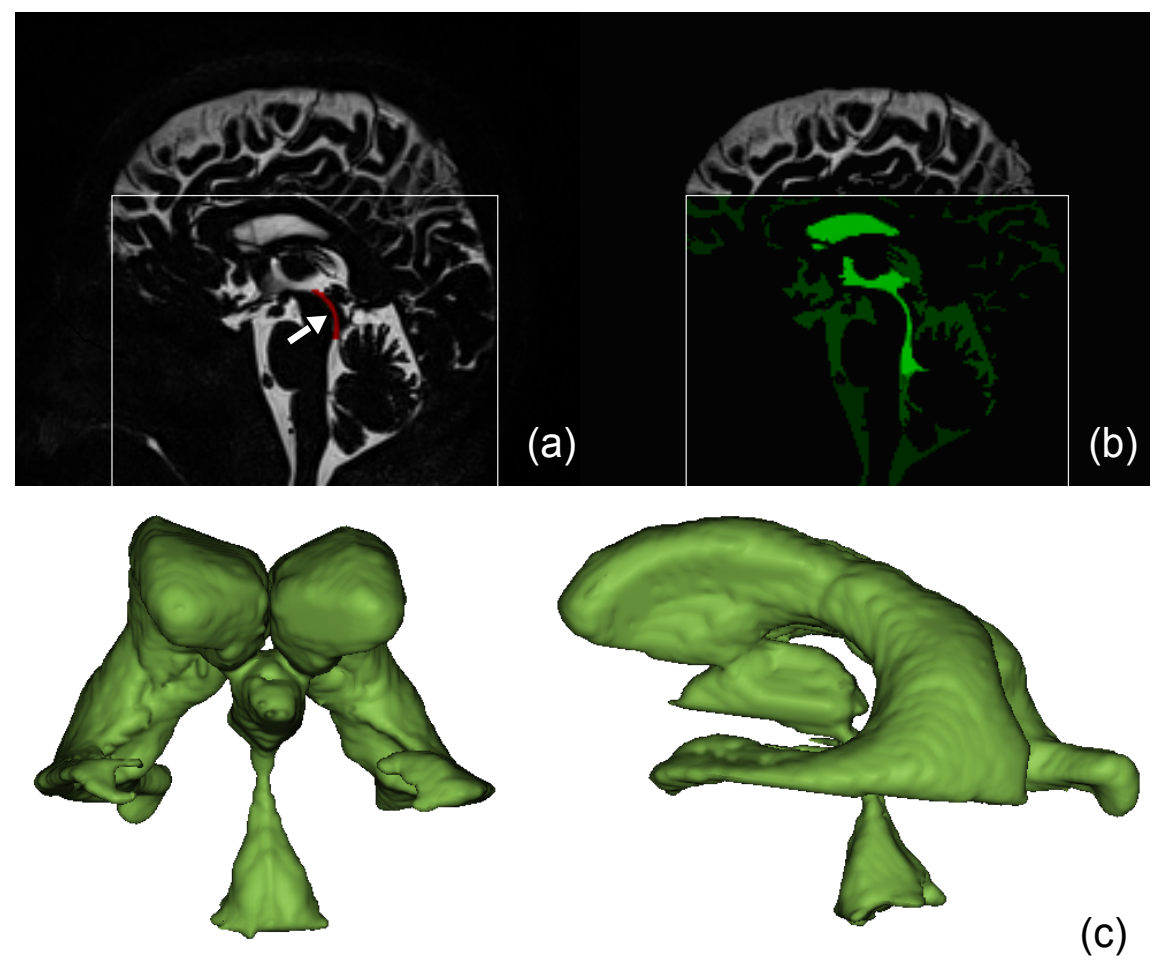

Figure 6: Ventricular space retrieving using a grayscale reconstruction by dilation of the cerebral aqueduct inside the original image: (a) the initial image (the mask) and the cerebral aqueduct (the marker) colored in red; (b) result of the grayscale reconstruction with regional maxima colored in different tons of green; (c) frontal (left) and sagittal (right) views of the ventricular space for an healthy adult using a grayscale reconstruction by dilation of the cerebral aqueduct inside the region of interest $\mathcal{R}$.

subset, as well as a linear discriminant analysis on the clinical dataset distribution to classify healthy and pathological cases.

\subsection{Volume assessment}

Experiments for volume assessment were carried out on 5 phantoms of known volume (image size: 128 slices of $250 \times 250$ in the sagittal plane) and 38 clinical images (image size: 160 slices of around $260 \times 790$ in the sagittal plane). Processing took place on a conventional PC (Intel Pentium Dual Core $1.60 \mathrm{GHz} / 4$ GB main memory, Linux 2.6 operating system). The overall time to assess volumes is $20 \mathrm{~min}$ or less for each patient: $12 \mathrm{~min}$ or less for the image acquisition and approximately $6 \mathrm{~min}$ for its processing. Obviously, this computation time could be dramatically reduced by using a computer with an architecture dedicated to three-dimensional image processing.

\subsubsection{Images acquisition}

Magnetic resonance images were acquired in the sagittal plane on a $1.5 \mathrm{~T}$ system (Magnetom Avanto; Siemens Medical Solutions, Erlangen, Germany). The magnetic resonance 

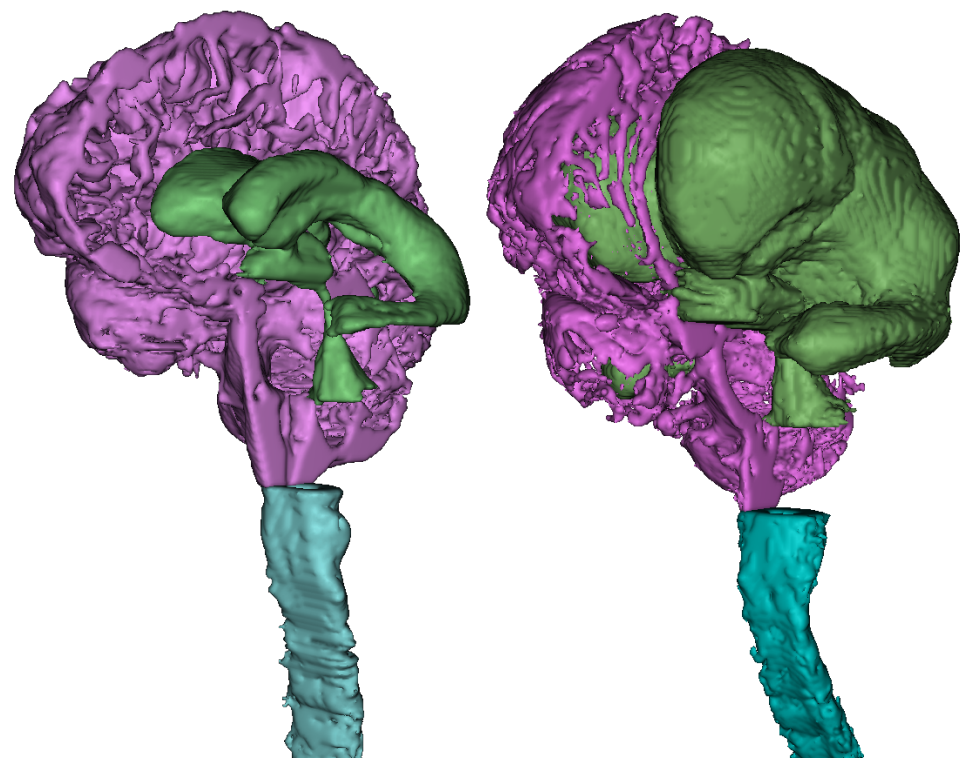

Figure 7: Partial three-dimensional surface rendering (the cortical subarachnoid space was cut along the sagittal plane). Ventricular, cortical subarachnoid and spinal subarachnoid spaces are respectively colored in green, magenta and cyan. Left: an healthy adult; right: a non-communicating hydrocephalus patient.

sequence in [7], called SPACE (Sampling Perfection with Application optimized Contrast using different flip-angle Evolution), is a variant of the T2-weighted turbo spin echo sequence with variable flip-angles. The sequence for clinical images was taken as follows: repetition time TR (ms)/echo time TE (ms), 2400/762; turbo factor of $141 ; 250 \times 250 \mathrm{~mm}$ field of view; $256 \times 256$ acquisition matrix; $1 \mathrm{~mm}$ isotropic resolution; number of excitations $1.4^{1} ; 160$ slices for head station that may be reduced to 70 slices for each spinal station to accelerate acquisition; acquisition time, 9 to 12 min according the heart rate.

\subsubsection{Entire volume assessment validation}

The segmentation method for the entire volume assessment was first validated on phantom images using the same sequence. Images were acquired from synthetic resin phantoms mimicking the intracranial brain sulci with various shapes and volumes as shown in Fig. 8. Our segmentation method on phantoms has resulted in an accuracy of $98.5 \% \pm 1.8$ against $94.6 \% \pm 4.0$ for the semi-manual segmentation by an expert using the method proposed in [7].

Clinical dataset images were evaluated by a physician expert. First the expert ensured that anatomical structures except for cerebrospinal fluid were correctly removed. Second, the accuracy of the segmentation process was assessed by comparing the fluid boundaries using the segmented images and the original images.

\footnotetext{
${ }^{1}$ Only central region of the $3 \mathrm{D} \mathrm{k}$-space was resampled for the second time. This second acquisition only covered $40 \%$ of the entire k-space.
} 


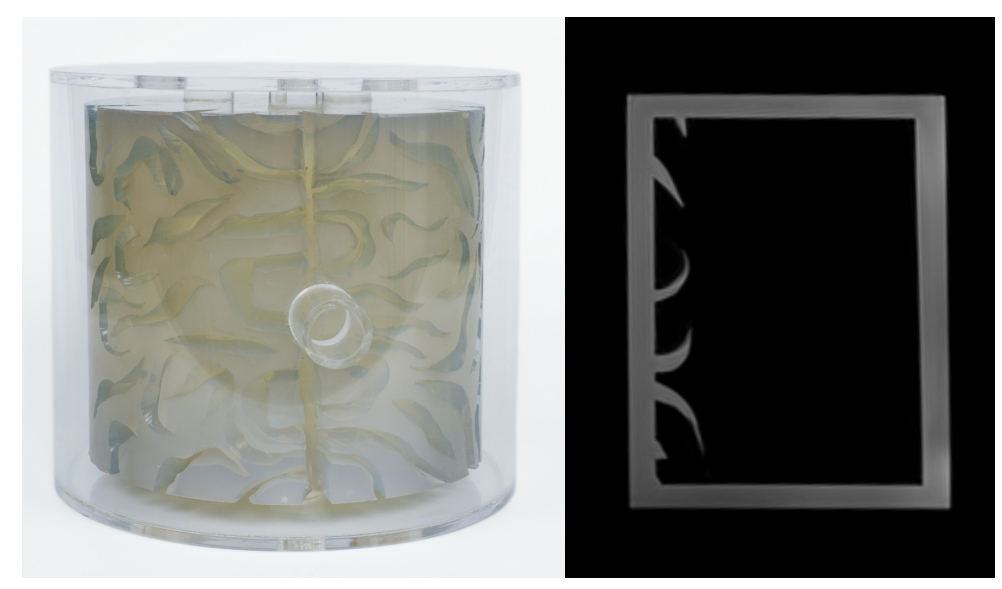

Figure 8: Photography of a synthetic resin phantom mimicking the brain sulci used to validate the entire cerebrospinal fluid volume segmentation method. Right: cross-section of the magnetic resonance image.

\subsubsection{Spaces volume assessment}

The obtained volumes of the cerebrospinal fluid spaces are summarized in Table 1 where they are matched with previous results from the literature $[3,4,5,6,7]$ for healthy adults, communicating and non-communicating hydrocephalus classes. In Table 1, results vary slightly depending on the articles. The reason is not only due to the difference of the methods, but also because of different data and image resolutions. Results on the spinal subarachnoid space confirms the assumption in [6] that the spinal subarachnoid volume assessment is important to discriminate between hydrocephalus patients, i.e. communicating and non-communicating hydrocephalus classes.

The expert ensured that the accuracy of the separation process was assessed by comparing the fluid boundaries using the segmented images and the native clinical images.

\subsection{Statistical analysis of volumes}

Clinical images were acquired from different subjects between 23 and 91 years old: 12 healthy volunteers and 26 patients among which 20 communicating hydrocephalus and 6 non-communicating hydrocephalus. The software package $\mathrm{R}$ [25] for Linux workstations was used for statistical analysis.

\subsubsection{Linear regression for healthy adults}

Observing the distribution of the subarachnoid volume relative to the ventricular one has provided us the idea of applying a linear regression analysis to the healthy people subset. Let $V_{\mathrm{V}}$ and $V_{\mathrm{S}}$ the respective volumes of the ventricular and entire subarachnoid spaces. We use the linear model for fitting to our healthy people volume pair $\left(V_{\mathrm{V}}, V_{\mathrm{S}}\right)$ set: $V_{\mathrm{S}}=\beta_{0}+\beta_{1} V_{\mathrm{V}}+\epsilon$, and optimize the intercept $\beta_{0}$ and the slope $\beta_{1}$ by minimizing the residual error $\epsilon$. We obtain $\beta_{0}=0$ and $\beta_{1}=10.73$, namely:

$$
V_{\mathrm{S}}=10.73 V_{\mathrm{V}}
$$


Table 1: Comparison of our cerebrospinal fluid volumes with the previous results for healthy adult people, communicating $(\mathrm{CH})$ and non-communicating hydrocephalus patients $(\mathrm{NCH})$. Each value is represented by a mean \pm standard deviation.

\begin{tabular}{|c|c|c|c|c|}
\hline \multicolumn{5}{|c|}{ Cerebrospinal fluid volumes $\left(\mathrm{cm}^{3}\right)$} \\
\hline Method & Class & Ventricles & Cortical sub. & Spinal sub. \\
\hline \multirow{2}{*}{ Matsumae [3] } & Controls & $17 \pm 9$ & $89 \pm 27$ & - \\
& CH & $116 \pm 42$ & $133 \pm 42$ & - \\
& NCH & $253 \pm 96$ & $172 \pm 63$ & - \\
\hline \multirow{2}{*}{ Tsunoda [4] } & Controls & $24 \pm 10$ & $177 \pm 57$ & - \\
\hline Palm [5] & CH & $76 \pm 19$ & $203 \pm 56$ & - \\
\hline \multirow{2}{*}{ Hodel [7] } & CH & $156 \pm 46$ & $201 \pm 37$ & - \\
& CH & $199 \pm 56$ & $374 \pm 110$ & - \\
\hline Edsbagge [6] & NCH & $397 \pm 165$ & $234 \pm 133$ & - \\
\hline \multirow{2}{*}{ The proposed method } & Controls & - & - & $81 \pm 13$ \\
& CH & $154 \pm 58$ & $269 \pm 62$ & $65 \pm 18$ \\
& NCH & $340 \pm 127$ & $154 \pm 59$ & $99 \pm 27$ \\
\hline
\end{tabular}

Table 2: Optimal linear regression model on the healthy adult subset from the clinical data set.

\begin{tabular}{llllll}
\hline Model & $R^{2}$ & $\sigma^{2}$ & Standard Error & $F$ & $p$-value \\
\hline$V_{\mathrm{S}}=10.73 V_{\mathrm{V}}$ & 0.8883 & 10.24 & 0.0088 & 87.51 & $1.43 \mathrm{e}-06$ \\
\hline
\end{tabular}

Table 2 includes some statistics about the model: 1) the coefficient of determination $R^{2}(=88.83 \%)$ indicates that the predictor explains rather well the answer with $95 \%$ of significance; 2$)$ the Fischer's $F$-statistic $(=87.51)$ and the $p$-value $(=1.43 \mathrm{e}-06 \ll 0.05)$ show further that the null hypothesis $\left(\beta_{1}=0\right)$ can be rejected with a strong presumption, and that our model is significant. In addition, the graphical residuals analysis reinforces the linear regression assumptions (Fig. 9). Figure 10 shows the distribution of volumes of the entire subarachnoid and ventricular spaces with the model obtained from (5).

This linear regression analysis indicates that the ratio of the entire subarachnoid space volume to the ventricular space volume is a proportionality constant equal to 10.73 for healthy adults. This proportionality constant ensures a steady-state intracranial pressure.

\subsubsection{Classification of clinical data}

A linear discriminant analysis [26] was performed on the clinical dataset using volumes of the entire subarachnoid and ventricular spaces as the input variables. We consider three classes: "healthy adults, communicating hydrocephalus and non-communicating hydrocephalus". Let $T P, F P, T N$ and $F N$ the respective numbers of true positives, false positives, true negatives and false negatives. The sensitivity $S_{\mathrm{e}}$ and the specificity (or recall) $S_{\mathrm{p}}$ are respectively defined as $S_{\mathrm{e}}=T P /(T P+F N)$ and $S_{\mathrm{p}}=T N /(T N+F P)$. The additional 

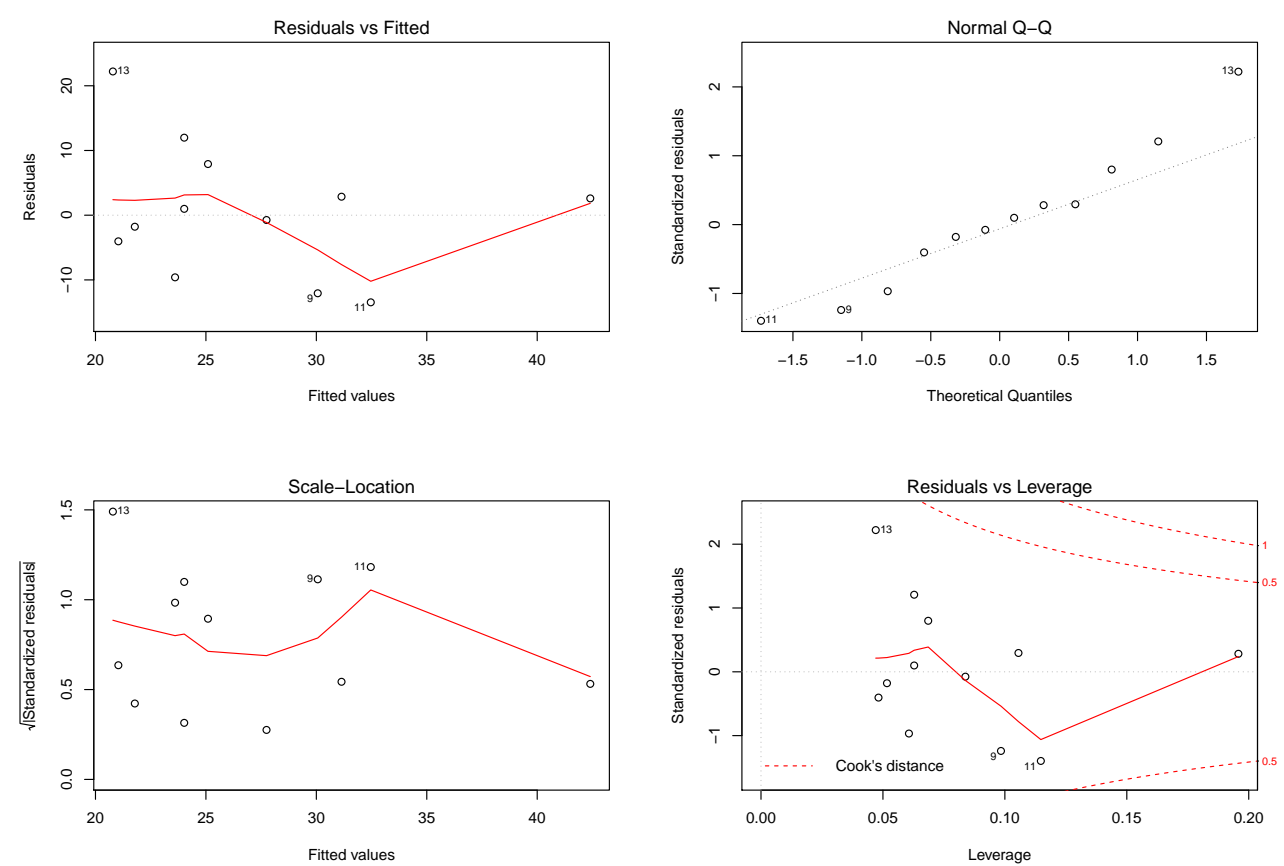

Figure 9: Residual plots from the linear regression on clinical data.

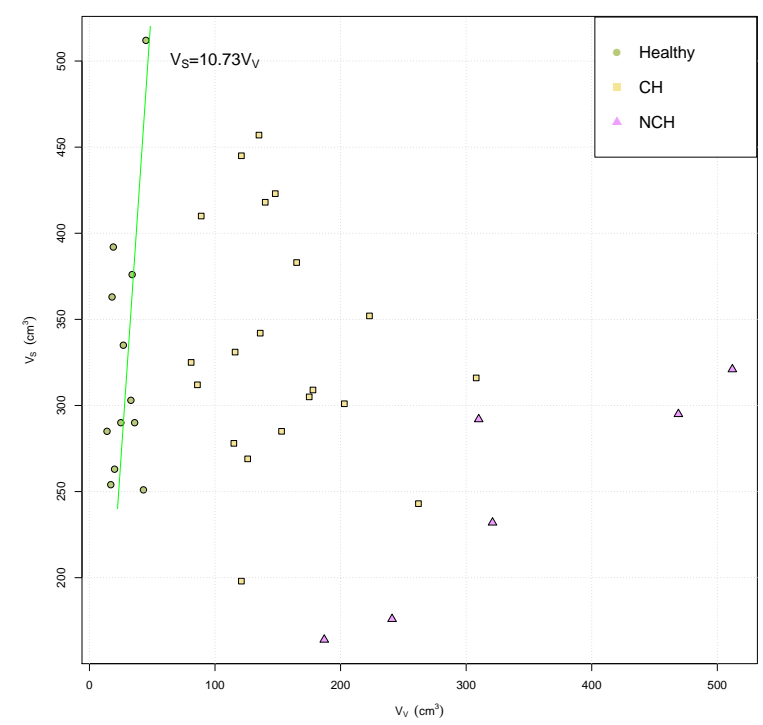

Figure 10: Distribution of the entire subarachnoid space volume $V_{\mathrm{S}}$ relative to the ventricular volume $V_{\mathrm{V}}$ of the clinical dataset. Note the resulting linear regression between volumes of the entire subarachnoid and ventricular spaces for the healthy adults subset such that: $V_{\mathrm{S}}=10.73 V_{\mathrm{V}}$. 


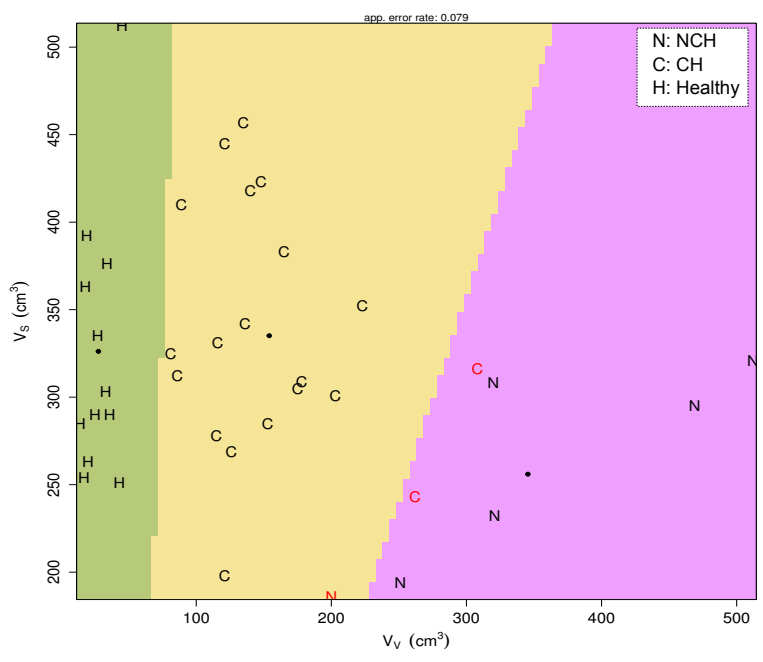

Figure 11: Resulting partition plot on clinical data by using a linear discriminant analysis considering three classes: "healthy $(\mathrm{H})$, communicating hydrocephalus $(\mathrm{C})$ and non-communicating hydrocephalus (N)".

$F_{1}$-score metric is also used to validate the classifier. It is defined as $F_{1}=2 p S_{\mathrm{e}} /\left(p+S_{\mathrm{e}}\right)$ where $p$ stands for the precision, defined by $p=T P /(T P+F P)$.

The predicted classification between healthy adults and pathological cases results in a sensitivity $S_{\mathrm{e}}$ of $100 \%$, a specificity $S_{\mathrm{p}}$ of $100 \%$ and a $F_{1}$-score of $100 \%$, which is efficient to discriminate between classes, especially to distinguish elderly healthy adults from elderly patients with communicating hydrocephalus. The predicted classification of noncommunicating hydrocephalus results in a sensitivity $S_{\mathrm{e}}$ of $83.33 \%$, a specificity $S_{\mathrm{p}}$ of $91.66 \%$ and a $F_{1}$-score of $76.92 \%$, which is a suitable result to suspect a non-communicating hydrocephalus (Fig. 11). By considering the spinal subarachnoid volume only, the predicted classification of non-communicating hydrocephalus results in a sensitivity $S_{\mathrm{e}}$ of $83.33 \%$, a specificity $S_{\mathrm{p}}$ of $95.24 \%$ and a $F_{1}$-score of $90.90 \%$, which may show some interest of the spinal subarachnoid volume assessment to suspect a non-communicating hydrocephalus; this remains to be confirmed with a larger number of data.

\section{Conclusion and perspectives}

This work uses a new whole body magnetic resonance imaging sequence that significantly highlights the cerebrospinal fluid, and succeeds in providing a volume assessment of the fluid spaces. Volumes are automatically retrieved through segmentation and separation steps, which use image properties, anatomical and geometrical features, as well as a topological assumption on the entire fluid shape. We made statistical analyses on entire subarachnoid and ventricular volumes. Our results show that the ratio of the entire subarachnoid space volume to the ventricular space volume is a proportionality constant $(=10.73)$ for healthy adults in order to maintain a stable intracranial pressure. Indeed, communicating hydro- 
cephalus patients have ratios in the range [0.93, 4.61] and non-communicating hydrocephalus patients have ratios in the range $[0.63,0.93]$. This result may confirm that this ratio can be an efficient functional index to distinguish healthy adult people from pathological cases, especially to distinguish elderly healthy adults from elderly patients with communicating hydrocephalus.

As the database is currently in expansion, our tool facilitates the refinement of these results in future work. In addition, it allows to study the variation of cerebrospinal fluid volumes before and after surgery on a patient suffering from hydrocephalus, and the evolution of these volumes as a function of age.

This new ability to assess volumes of the cerebrospinal fluid spaces, which is a main parameter for the diagnosis, for the treatment decisions, for the monitoring of patients, and finally for clinical research, may represent an important breakthrough in the field of computer-aided neuro-imaging.

\section{Conflict of interest statement}

The authors have no actual or potential conflict of interest including any financial, personal, or other relationships with other people or organizations to disclose.

\section{References}

[1] Sakka, L., Coll, G., Chazal, J.. Anatomy and physiology of cerebrospinal fluid. European Annals of Otorhinolaryngology, Head and Neck Diseases 2011;128(6):309-316.

[2] Rekate, H.L.. A consensus on the classification of hydrocephalus: its utility in the assessment of abnormalities of cerebrospinal fluid dynamics. Child's Nervous System 2011;27(10):1535-1541.

[3] Matsumae, M., Kikinis, R., Mórocz, I., Lorenzo, A.V., Albert, M.S., Black, P.M., et al. Intracranial compartment volumes in patients with enlarged ventricles assessed by magnetic resonance-based image processing. Journal of Neurosurgery 1996;84(6):972-981.

[4] Tsunoda, A., Mitsuoka, H., Sato, K., Kanayama, S.. A quantitative index of intracranial cerebrospinal fluid distribution in normal pressure hydrocephalus using an MRI-based processing technique. Neuroradiology 2000;42(6):424-429.

[5] Palm, W., Walchenbach, R., Bruinsma, B., Admiraal-Behloul, F., Middelkoop, H., Launer, L., et al. Intracranial compartment volumes in normal pressure hydrocephalus: Volumetric assessment versus outcome. AJNR American Journal of Neuroradiology 2006;27(1):76-79.

[6] Edsbagge, M., Starck, G., Zetterberg, H., Ziegelitz, D., Wikkelso, C.. Spinal cerebrospinal fluid volume in healthy elderly individuals. Clinical Anatomy 2011;24(6):733-740.

[7] Hodel, J., Silvera, J., Bekaert, O., Rahmouni, A., Bastuji-Garin, S., Vignaud, A., et al. Intracranial cerebrospinal fluid spaces imaging using a pulse-triggered three-dimensional turbo spin echo MR sequence with variable flip-angle distribution. European Radiology 2011;21(2):402-410.

[8] Lebret, A., Petit, E., Durning, B., Hodel, J., Rahmouni, A., Decq, P.. Quantification of the cerebrospinal fluid from a new whole body MRI sequence. In: van Ginneken, B., Novak, C.L., editors. SPIE Medical Imaging 2012: Computer-Aided Diagnosis; vol. 8315. 2012, p. 83153H-83153H-7.

[9] Klette, R., Rosenfeld, A.. Digital Geometry: Geometric Methods for Digital Picture Analysis. San Francisco: Morgan Kaufmann; 2004.

[10] Ségonne, F.. Segmentation of medical images under topological constraints. Ph.D. thesis; Massachusetts Institute of Technology; Cambridge MA, USA; 2005.

[11] Bazin, P.L., Pham, D.L.. Topology-preserving tissue classification of magnetic resonance brain images. IEEE Transactions on Medical Imaging 2007;26(4):487-496. 
[12] Miri, S., Passat, N., Armspach, J.P.. Topology-preserving discrete deformable model: Application to multi-segmentation of brain MRI. In: 3rd International Conference on Image and Signal Processing (ICISP 2008); vol. 5099 of Lecture Notes in Computer Science. Berlin, Heidelberg: Springer-Verlag; 2008, p. $67-75$.

[13] Rueda, A., Acosta, O., Couprie, M., Bourgeat, P., Fripp, J., Dowson, N., et al. Topology-corrected segmentation and local intensity estimates for improved partial volume classification of brain cortex in MRI. Journal of Neuroscience Methods 2010;188(2):305-315.

[14] Tsai, W.H.. Moment-preserving thresholding: a new approach. Computer Vision, Graphics, and Image Processing 1985;29(3):377-393.

[15] Shapiro, L.G., Stockman, G.C.. Computer Vision. Prentice-Hall; 2001.

[16] Zack, G.W., Rogers, W.E., Latt, S.A.. Automatic measurement of sister chromatid exchange frequency. Journal of Histochemistry and Cytochemistry 1977;25(7):741-753.

[17] Saito, T., Toriwaki, J.I.. New algorithms for euclidean distance transformation on an N-dimensional digitized picture with applications. Pattern Recognition 1994;27(11):1551-1565.

[18] Bertrand, G., Malandain, G.. A new characterization of 3-dimensional simple points. Pattern Recognition Letters 1994;15:169-175.

[19] Lesage, D., Angelini, E.D., Bloch, I., Funka-Lea, G.. A review of 3D vessel lumen segmentation techniques: Models, features and extraction schemes. Medical Image Analysis 2009;13(6):819-845.

[20] Vincent, L.. Morphological grayscale reconstruction in image analysis: Applications and efficient algorithms. IEEE Transactions on Image Processing 1993;2(2):176-201.

[21] Sato, Y., Nakajima, S., Atsumi, H., Koller, T., Gerig, G., Yoshida, S.. Three-dimensional multiscale line filter for segmentation and visualization of curvilinear structures in medical images. Medical Image Analysis 1998;2(2):143-168.

[22] Frangi, A.F., Niessen, W.J., Vincken, K.L., Viergever, M.A.. Multiscale vessel enhancement filtering. In: W.M. Wells, A.C., Delp, S., editors. 1st international conference on Medical Image Computing and Computer-Assisted Intervention (MICCAI '98); vol. 1496 of Lecture Notes in Computer Science. Berlin, Heidelberg: Springer-Verlag; 1998, p. 130-137.

[23] Sethian, J.A.. Level Set Methods and Fast Marching Methods: Evolving Interfaces in Computational Geometry, Fluid Mechanics, Computer Vision, and Materials Science. Cambridge University Press; 1999.

[24] Cohen, L.D.. On active contours models and balloons. Computer Vision, Graphics, and Image Processing: Image Understanding 1991;53(2):211-218.

[25] R Development Core Team, . R: A Language and Environment for Statistical Computing. R Foundation for Statistical Computing; Vienna, Austria; 2011.

[26] Duda, R.O., Hart, P.E., Stork, D.G.. Pattern Classification. Wiley Interscience; 2nd ed.; 2000. 